\title{
Intervención sobre problemas de conducta tempranos como prevención indicada del consumo de drogas: Siete años de seguimiento
}

\author{
Intervention on early-onset conduct problems as indicated \\ prevention for substance use: A seven-year follow up
}

\author{
estrella Romero*; Concepción Rodríguez*; Paula Villar*; X. Antón Gómez-Fraguela*. \\ *Departamento de Psicología Clínica y Psicobiología. \\ Universidad de Santiago de Compostela.
}

\section{Resumen}

Este trabajo tiene como objetivo evaluar los efectos a largo plazo de un programa manualizado que interviene sobre niños con problemas de conducta, sus familias y sus profesores. El programa involucró, durante el curso 2007-08, a 14 escuelas que fueron asignadas aleatoriamente a las condiciones de intervención (45 familias participantes) y control (30 familias). A partir de un screening que identificó niños con problemas significativos de conducta en la familia y en la escuela, el programa fue aplicado en ocho de esos centros. Siete años más tarde, se pudo contactar de nuevo con 58 familias (37 de intervención y 21 control), con características equivalentes al total de participantes en el estudio inicial. Por medio de informes obtenidos de los participantes y de sus padres, y a través de comparaciones con análisis multivariables de la varianza, se apoya la eficacia del programa en la reducción de los problemas de conducta y de la implicación con amigos antisociales. Asimismo, se encuentra que el programa promueve la competencia social y comunicativa de los participantes. En cuanto al consumo de drogas, el grupo de intervención muestra actitudes menos favorables hacia las drogas, menor intención de consumo, menor frecuencia de consumo de tabaco y menor intensidad de consumo de alcohol. Estos resultados apoyan la utilidad de los programas multicomponente de intervención sobre los problemas de conducta como una vía para prevenir, a largo plazo, trayectorias de desarrollo desfavorables, en las que el consumo de drogas es una pieza fundamental.

Palabras clave: Prevención indicada; Problemas de conducta; Niñez; Consumo de drogas.

\section{Abstract}

The aim of this study is to evaluate the long-term effects of a manualised program which intervenes on children with earlyonset conduct problems, their families and teachers. The program evaluation involved 14 primary schools which were randomly assigned to the intervention (45 participating families) and control (30 families) conditions during 2007-2008. After a screening process which identified children with significant conduct problems both at home with their family and at school, the program was implemented in eight schools. Seven years later, 58 families ( 37 from the intervention group and 21 from the control group), with characteristics equivalent to those of the study's entire initial group, were contacted again. With measures administered to the children and their parents, comparisons through multivariate analyses of variance between intervention and control groups supported the program's efficacy in reducing both conduct problems and relations with antisocial peers. Furthermore, the program fostered social and communication skills. As regards drug use, the intervention group showed less favourable attitudes towards drugs, lower intention of drug use, lower frequency of tobacco use and lower intensity of alcohol use. These results support the usefulness of multicomponent programs for conduct problems as a way to prevent, in the long term, unfavourable developmental trajectories, where drug use is a key element.

Keywords: Indicated prevention; Conduct problems; Childhood; Substance use. 


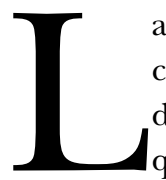

a prevención del consumo de drogas ha sido un campo ampliamente desarrollado en las últimas décadas (Scheier, 2015). Sin embargo, a pesar de que se ha invertido un enorme caudal de esfuerzos en lo que Gordon (1983) denominó "prevención universal”, la prevención "indicada” (dirigida específicamente a individuos que manifiestan indicadores que permiten anticipar un problema; véase Foxcrot, 2014) es un campo menos desarrollado. En los últimos años, desde diferentes agencias, tanto estadounidenses (NIDA, 2003) como europeas (EMCDDA, 2009), se ha reclamado la necesidad de ampliar los recursos de prevención, con especial atención a aquellos individuos que presentan, tempranamente, un riesgo de patrones de consumo más severo. En este sentido, la investigación ha focalizado su interés en los problemas de conducta tempranos, como un indicador clave para predecir el abuso de drogas y otros problemas comórbidos, tales como comportamientos antisociales, desajuste escolar y dificultades emocionales.

El estudio de los problemas de conducta perturbadora (o problemas "externalizantes") dentro de los determinantes del consumo de drogas ha experimentado un fuerte impulso en los últimos años (e.g., Martel et al., 2009; Sitnick, Shaw y Hyde, 2014) La psicopatología del desarrollo (Cicchetti y Cohen, 2006) subraya la necesidad de ir más allá de los determinantes inmediatos, para conocer cómo se van desarrollando, desde la niñez, las trayectorias de alto riesgo para el abuso de drogas. En este sentido, recientemente se han propuesto modelos teóricos de "cascada" (Dodge et al., 2010; Haller, Handley, Chassin y Bountress, 2010), que sitúan a los problemas de conducta en el centro de un proceso acumulativo, donde, progresivamente, se van generando más dificultades. Así, los problemas de conducta, a través de cadenas de influencia recíproca con dificultades familiares, rechazo por parte de otros niños, desajuste escolar y limitadas habilidades de autocontrol y de procesamiento emocional, generan efectos tipo "bola de nieve", de forma que las oportunidades para un desarrollo saludable se van reduciendo. En este contexto, los problemas de conducta se cronifican y se va consolidando un estilo de vida desajustado donde emerge un consumo problemático de drogas, junto con alteraciones de tipo antisocial, impulsivo y emocional (Webster-Stratton y Hammond, 1997).

En apoyo a estos modelos, la investigación ha mostrado que los problemas de conducta se asocian consistentemente con indicadores de severidad en el consumo, incluyendo altas tasas de poliadicción, alta implicación en actividades delincuentes, alto riesgo de abandono del tratamiento, y mala respuesta a las intervenciones (Hawkins, 2009; Hser, Grella, Collins y Teruya, 2003). Por ello, los programas que abordan los problemas de conducta tempranos son consideradas herramientas necesarias para una prevención del uso problemático de drogas desde la niñez (EMCDDA, 2009; Glantz, 2002).
Dada la amplitud y la complejidad de factores que intervienen en la etiología y el desarrollo de los problemas de conducta, la investigación previa ha reiterado la necesidad de programas multicomponente, que intervengan al mismo tiempo sobre diferentes fuentes de riesgo (Conduct Problem Prevention Research Group, 2004; Foster, Olchowski y Webster-Stratton, 2007). Aunque en la literatura previa se encuentran múltiples ejemplos de programas para la intervención sobre problemas de conducta, las bases de datos de programas de prevención basados en la evidencia (e.g., Blueprints for Youth Health Prevention, Center for Substance Abuse Prevention) identifican un número muy reducido de programas multicomponente, dirigidos a niños con comportamiento perturbador, que hayan sido sometidos a evaluación a largo plazo evidenciando efectos sobre el consumo de drogas. Los programas Coping Power (Zonnevylle-Bender, Matthys, Van De Wiel y Lochman, 2007) o Linking the Interests of Families and Teachers (Eddy, Reid, Stoolmiller y Petrow, 2003) son citados como ejemplos de intervenciones que, actuando sobre varios ámbitos psicosociales, y enfocados hacia los problemas de conducta, han mostrado efectos sobre el comportamiento posterior de los jóvenes, incluyendo prevención del uso de sustancias.

Asimismo, la revisiones sobre este tema han llamado la atención de sobre la necesidad de avanzar en la investigación sobre el efecto preventivo de la intervención en niños con problemas emocionales y conductuales (Salvo et al., 2012). Por todo ello, en este estudio se realiza una evaluación, siete años después de su aplicación, de un programa multicomponente destinado a la intervención sobre niños con problemas de conducta de inicio temprano. El programa EmPeCemos (vease Romero, Villar, Luengo y Gómez-Fraguela, 2009; http://www.emcdda.europa.eu/html.cfm/index52035EN. html?project_id=ES_03\&tab=overview) se asienta en los modelos "cascada" planteados por la psicopatología del desarrollo (Dodge et al., 2010; Granic y Patterson, 2006) y ofrece una intervención manualizada y coordinada sobre el ámbito familiar, el profesorado y los propios niños. Estudios previos sobre la eficacia de cada uno de sus componentes y sobre el programa completo, mostraron que la intervención permite reducir los problemas de conducta (incluyendo hiperactividad y conductas desafiantes), con tamaños del efecto $d$ que oscilaron entre .72 y .78 para las medidas globales de problemas de conducta, y que sus efectos se sostienen a lo largo de un año (Robles, 2009; Romero, Villar, Luengo, Gómez-Fraguela y Robles, 2014). El programa además muestra efectos significativos, a corto plazo, sobre las variables conceptualmente mediadoras: prácticas parentales, habilidades socioemocionales de los niños y autoeficacia del profesorado para el manejo de las conductas perturbadoras (Romero, Villar y Gómez-Fraguela, 2010), de acuerdo con los objetivos inmediatos establecidos para el programa.

El objetivo del presente estudio es evaluar el programa a largo plazo, examinando sus efectos cuando los participan- 
tes se encuentran en la adolescencia. Teniendo en cuenta los planteamientos de la psicopatología del desarrollo, y teniendo en cuenta el papel de los problemas de conducta tempranos en el desarrollo del consumo de drogas, se atenderá no sólo a los patrones de conductas disruptivas, sino también al consumo de sustancias de los jóvenes.

\section{Métodos}

\section{Participantes}

La selección de los participantes se llevó a cabo en el curso académico 2007-2008, a través de un screening realizado en 14 centros de educación primaria en Galicia, que fueron seleccionados aleatoriamente en el área geográfica próxima a Santiago de Compostela. Para este screening se utilizó un instrumento de 10 ítems basado en el Teacher Report Form (TRF, Achenbach, 1991a) de acuerdo con las indicaciones de programas previos dirigidos a niños con problemas de conducta (Larson y Lochman, 2002). Este instrumento fue cumplimentado por los profesores tutores para la evaluación de los niños de los cursos segundo a cuarto. Ante los casos que el screening permitía identificar como susceptibles de intervención, se hizo una evaluación detallada atendiendo a la información de los padres (a través del CBCL de Achenbach, 1991b) y de profesores (a través de la versión completa del TRF) A partir de esta evaluación, se seleccionaron las familias cuyos niños presentaban problemas de conducta significativos (puntuaciones $\mathrm{T}$ mayores a 70 en la dimensión "Externalizante") tanto en el ámbito familiar como escolar. Fueron criterios de exclusión la presencia de un diagnóstico de retraso mental o trastorno generalizado del desarrollo. De las 88 familias seleccionadas, accedieron a participar en el estudio 75. De los 14 centros educativos participantes, se asignaron aleatoriamente 8 a la condición "intervención" (con un total de 45 niños con problemas de conducta; media de edad: 8.34) y 6 a la condición "control" (un total de 30 niños con problemas de conducta: 8.27 ). No se encontraron diferencias significativas entre ambos grupos en variables sociodemográficas básicas (edad, género, composición familiar) ni en el nivel de problemas de conducta.

El programa multicomponente fue aplicado en los 8 centros del grupo de intervención durante los cursos académicos 2007-2008 (programa base) y 2008-2009 (módulos de apoyo). En 2014, con el fin de realizar este seguimiento a largo plazo, se inició un nuevo contacto con los participantes. De las 75 familias participantes en el estudio inicial, se pudo contactar con 64. Las dificultades de contacto con las familias restantes tienen que ver con cambios de teléfono y/o de domicilio. De las 64 familias con las que se contactó, 58 accedieron a participar. Las negativas obtenidas se correspondieron, en cuatro casos, a limitaciones de tiempo y/o dificultades transitorias de salud; en otros dos casos, las familias se negaron a participar sin proporcionar explica- ciones específicas. El porcentaje de atrición fue, por tanto, de un $22 \%$ en el período de siete años; particularmente, en el grupo de intervención la pérdida de casos fue de un 17\% (participaron 37 de 45 familias), y en el grupo control, como era esperable, la implicación de los participantes fue menor (participaron 21 de 30 familias; 30\% de atrición). Así pues, la muestra que participa en este estudio de evaluación está conformada por 58 jóvenes ( 56 chicos y 2 chicas) que, en el momento del seguimiento, presentan una media de edad de 15.25 años.

\section{Descripción de la intervención}

El programa EmPeCemos consta de tres componentes. El programa de familias (12 sesiones) entrena a los padres en habilidades para establecer relaciones positivas con los niños, promover comportamientos positivos en ellos y enfrentarse a las conductas problemáticas. El programa incluye además módulos de entrenamiento en habilidades de autocontrol, resolución de problemas y comunicación, como apoyo a las estrategias parentales que necesitan aprenderse, y como apoyo, además, al desarrollo cognitivo-emocional de los niños. El programa de niños consta también de 12 sesiones que entrenan los niños con problemas de conducta en habilidades para el reconocimiento de las emociones, el autocontrol, la resolución de problemas y la toma de perspectivas, y las habilidades sociales (una descripción detallada del programa puede encontrarse en Romero et al., 2009). Finalmente, el programa de profesores (8 sesiones), entrena en estrategias de manejo del comportamiento perturbador, al tiempo que potencia la colaboración con la familia y la estimulación del comportamiento ajustado en el entorno escolar. Seis meses y un año después de este programa "base" se aplican módulos de apoyo (dos sesiones para padres y niños y una sesión para profesores).

Los tres componentes del programa se aplican en grupos (de entre 5 y 10 participantes) utilizando técnicas basadas en el aprendizaje social: instrucción, discusión, modelado (en vivo y audiovisual), role-playing y, sobre todo, práctica guiada en el entorno natural. Los componentes se aplican simultánea y coordinadamente por parte de terapeutas previamente entrenados, con el fin de lograr cambios coherentes en los niños y en su entorno.

El programa multicomponente fue aplicado en las instalaciones de los centros educativos. Las tasas de participación en el programa fueron muy altas, especialmente considerando que el programa implica a participantes en alto riesgo de desajuste social. Las tasas de abandono del principio al final del programa base fueron de $8 \%$ para los componentes de padres y niños (4 de 45) y algo más elevadas (un 15\%; 19 de 125) para el componente de profesores. La asistencia a las sesiones también fue alta: como promedio, los padres asistieron a 9.24 sesiones, 10.43 para los niños (en ambos casos, con un máximo posible de 12), y 5.05 (máximo 8) para los profesores. Por otra parte, el seguimiento del proceso de 
implementación mostró que se aplicaron un promedio de un $88 \%$ (70 de 79 ) de las actividades propuestas en el componente de familias; un $76 \%$ (59 de 77) de las propuestas para niños y un $73 \%$ (30 de 41) de las propuestas para profesores. Esto avala la integridad en la aplicación del programa, que, además, fue aplicado con un alto grado de fidelidad a la racionalidad y los fundamentos del programa, tal y como pudo ser comprobado a través de los diarios de implantación, la plataforma virtual de seguimiento que se estableció y los cuestionarios de auto y hetero-evaluación para terapeutas cumplimentados en cada sesión.

Finalmente, los abandonos desde el principio de la intervención hasta el final de los módulos de apoyo fueron de $11 \%$ ( 5 de 45 ) para los componentes de padres y niños y de un $25 \%$ (31 de 125) de los profesores. De las sesiones de apoyo, a los seis meses se aplicó, como promedio, el $90 \%$ de las actividades previstas para las sesiones de padres (9 de 10), el $100 \%$ de las previstas para niños (9 de 9 ) y el $75 \%$ de las actividades previstas para profesores (3 de 4). El los módulos de apoyo a un año, se aplicaron, como promedio el $100 \%$ de las actividades previstas para padres (10 de 10), el $100 \%$ de las actividades previstas para niños (10 de 10) y el $66 \%$ de las actividades previstas para profesores (2 de 3 ).

\section{Instrumentos}

Para esta evaluación se tuvieron en cuenta datos recogidos a través de autoinformes y de escalas de calificación cumplimentadas por los padres.

\section{Instrumentos tipo autoinforme}

Consumo de drogas. El Cuestionario de Consumo de Drogas (Luengo, Romero, Gómez-Fraguela, Garra y Lence, 1999) incluye un amplia variedad de indicadores relacionados con el uso de sustancias, incluyendo actitudes, intenciones de consumo, edad de inicio, frecuencia y cantidad de consumo. El instrumento fue utilizado en múltiples estudios previos y ha mostrado su eficacia para evaluar los patrones de consumo de drogas en los jóvenes (e.g., Luengo, Villar, Sobral, Romero y Gómez-Fraguela, 2009; Sobral, Gómez-Fraguela, Romero, Luengo y Villar, 2012).

Problemas de conducta perturbadora. El Cuestionario de Conducta Antisocial (CCA; Luengo, Otero, Romero, Gómez-Fraguela y Tavares, 1999), permitió evaluar, a través de 37 ítems, la implicación de los jóvenes en conductas antisociales durante los últimos 12 meses. Una evaluación específica de los patrones de conducta agresiva se realizó a través de la Escala Autoinformada de Agresión Reactiva/Proactiva (Dodge y Coie, 1987), una escala breve (seis items) que permite evaluar la implicación en conductas agresivas premeditadas e instrumentales (agresión proactiva) y conductas agresivas emocionales como reacción a provocaciones reales o percibidas (agresión reactiva).

Adaptación escolar. La Escala de Adaptación Escolar de Berry, Phinney, Sam y Vedder (2006) incluye 8 items que ofrecen medidas de absentismo, rendimiento e implicación escolar.

Adaptación en el entorno grupal. Para examinar el grado de integración y adaptación en el entorno del grupo de iguales se utilizó, además de un ítem directo que pregunta por el número de amigos, la Escala de Confianza con los Amigos (del Inventory of Parent and Peer Atachment: IPPA; Armsden y Greenberg, 1987), que evalúa la cercanía y calidez en la relación con los iguales, y la Escala de Implicación con Amigos Antisociales (adaptada de Thornberry, Lizotte, Krohn, Farnworth y Jang, 1994), con cinco ítems que evalúan el contacto con amigos que realizan conductas antisociales.

Empatía. Dada la relevancia de la empatía y la toma de perspectivas como ingredientes de las competencias sociales necesarias para un desarrollo saludable, se incluyó un indicador autoinformado de empatía: la versión corta de la Escala de Empatía para Niños (Del Barrio, Aluja y García, 2004), compuesta por 10 ítems que evalúan la capacidad de los niños para verse afectados por los sentimientos de los demás.

Todos los instrumentos autoinformados habían sido utilizados ampliamente en estudios previos en nuestro contexto sociocultural, mostrando adecuadas propiedades psicométricas (e.g., López-Romero, Romero y Andershed, 2015).

\section{Escalas de calificación cumpimentadas por los padres/madres}

Problemas de conducta. Los padres/madres de los niños cumplimentaron el Child Behavior Checklist (CBCL; Achenbach, 1991b), que permite evaluar problemas externalizantes e internalizantes, y la Escala de Calificación de Conductas Disruptivas (Barkley, 1997), que permite obtener, a través de 26 ítems, medidas de dificultades atencionales, hiperactividad/impulsividad y conductas oposicionistas/desafiantes. Además, para la evaluacióna de patrones de conducta agresiva, se utilizó la versión para padres de la Escala de Agresión Reactiva/Proactiva (Dodge y Coie, 1987)

Competencias emocionales y sociales. La Escala de Competencia Social Fast Track; Conduct Problems Prevention Research Group, 1995) proporciona, a través de 12 ítems, medidas de Habilidades Prosociales/de Comunicación y Habilidades de Regulación Emocional.

Se pidió a las familias que estos instrumentos heteroinformados fuesen cumpimentados por la figura parental que compartiese más tiempo con el niño; en el $77 \%$ de los casos, la madre fue la fuente de información.

De nuevo, estos instrumentos habían sido ya adaptados y utilizados en estudios previos (López-Romero et al., 2015; Romero, Robles y Lorenzo, 2006), mostrando adecuadas propiedades psicométricas.

\section{Procedimiento}

En febrero 2014, siete años después de la aplicación del programa, se contactó telefónicamente o por carta con las 
familias que previamente habían participado en el estudio como parte del grupo de intervención o de control, para pedir su colaboración en este seguimiento. La aplicación de los instrumentos de evaluación se realizó entre los meses de abril y julio en las localidades de residencia de las familias, por parte de personal especializado que previamente no había estado implicado en la aplicación del programa. Si bien la participación en el estudio de apliación del programa no se habían utilizado incentivos, la participación en este seguimiento fue recompensada con 20 euros por cada familia participante. Todos los procedimientos fueron aprobados por el Comité de Bioética de la Universidad de Santiago de Compostela, y tanto el consentimiento informado de los padres como el asentimiento de los niños fueron obtenidos para participar en el estudio inicial y en el seguimiento.

\section{Resultados}

\section{Examen de la atrición}

En primer lugar se examinó en qué medida se había producido atrición diferencial en función de variables que a priori pueden considerarse relevantes para la valoración de los efectos del programa. En cuanto al grupo de intervención, no se encontraron diferencias significativas entre los participantes y los casos "perdidos", cuando se atiende a la edad (edad inicial de 8.42 en los participantes, frente a 8.38 en los casos perdidos; F=0.007, 1/43 gl, ns). En cuanto a los problemas de conducta previos, los casos perdidos tienden a mostrar puntuaciones más altas (media en puntuación global de conducta disruptiva en la escala de Barkley de 46.89, frente a 38.49 en los participantes), pero, con todo, estas diferencias no fueron estadísticamente significativas $(\mathrm{F}=2.05$, $1 / 42 \mathrm{gl}, \mathrm{ns})$. Sí se produjo atrición diferencial en función del género en el grupo de intervención. Aunque el número de niñas ya era pequeño ( 5 chicas, i.e. un $11 \%$ de la muestra total, reflejando la diferente prevalencia de los problemas de conducta tempranos entre niños y niñas), se pierden más chicas que chicos en el seguimiento (chi-cuadrado: 10.18, 1 $\mathrm{gl}, \mathrm{p}<.01)$.

En cuanto al grupo control, no se encontró atrición diferencial en función de la edad inicial (8.54 en los participantes, 8.21 en el grupo de casos perdidos, $\mathrm{F}=1.90,1 / 28 \mathrm{gl}$, ns), en problemas de conducta previos (aunque, de nuevo el grupo de casos perdidos tiende a mostrar más problemas previos, sin que estas diferencias sean estadísticamente significativas: 37.50 vs. $45.23, \mathrm{~F}=1.90,1 / 28 \mathrm{gl}$, ns). Tampoco hay pérdida diferencial de participantes en función del género (chi-cuadrado: 0.12, $1 \mathrm{gl}, \mathrm{ns}$ ).

Cuando, finalmente, dos grupos de participantes en el seguimiento (intervención y control) se comparan en esas variables básicas, no se observan diferencias significativas en el género (chi-cuadrado: 0.20, $1 \mathrm{gl}$, ns), en los problemas de conducta iniciales (38.19 en el grupo de intervención vs. 37.50 en el grupo control; F=0.03, 1/54 gl, ns) ni en la edad actual (15.06 en el grupo de intervención frente a 15.43 en el grupo control, $\mathrm{F}=1.25,1 / 55$, n.s.).

Así pues, el examen de la atrición sugiere que los participantes de intervención y control que finalmente pudieron ser seguidos forman grupos equivalentes a los que inicialmente participaron en el estudio. Esto apoya la validez interna de las comparaciones que se realizan en este estudio de seguimiento.

\section{Evolución diferencial de los problemas de conducta en el período de siete años}

Con el fin de conocer cómo progresan en ambos grupos los problemas de conducta disruptivos (medidos a través de la Escala de Calificación de Problemas Disruptivos de Barkley, 1997), en esta evaluación a largo plazo,se realizó un Análisis de Varianza 2x3 con una medida intrasujeto, representada por los momentos de evaluación T1 (pretest, previo a la aplicación del programa), T2 (postest, al finalizar el programa base) y T3 (seguimiento, siete años más tarde). De este modo se pretende identificar las trayectorias de evolución de los problemas de conducta a lo largo de los siete años de seguimiento, tomando una medida (la escala de Barkley) adecuada para su uso en los tres momentos del seguimiento. Los resultados muestran una interacción significativa entre la condición de tratamiento (intervención vs. control) y el tiempo ( $\mathrm{F}=8.70,2 / 45 \mathrm{gl}, \mathrm{p}<.001)$, lo cual indica que los problemas de conducta evolucionaron de forma diferente en ambos grupos. En la Figura 1 se representa esa evolución.

En la figura puede observarse el descenso en los problemas de conducta para el grupo de intervención entre el pretest y el postest, $\mathrm{t}(35)=6.58, \mathrm{p}<.001$, y se observa también que esa reducción se mantiene a lo largo del tiempo, sin diferencias significativas entre el postest y el seguimiento, $\mathrm{t}(34)=0.17$, ns. El grupo control, sin embargo, mantuvo altos niveles de problemas de conducta en el postest (sin diferencias significativas entre ambos puntos; $\mathrm{t}(19)=-.29$, ns, y, aunque la tendencia es descendente a lo largo del período de siete años, la diferencia

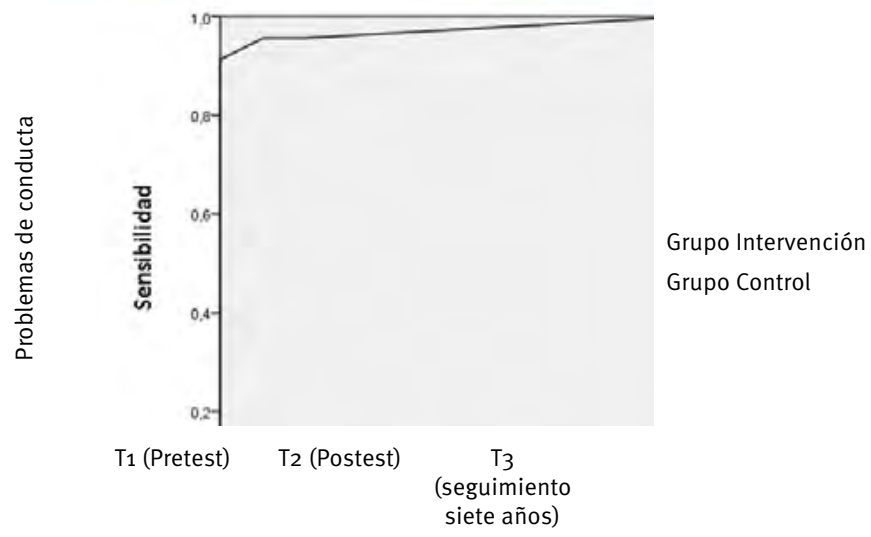

Figura 1. Evolución de los problemas de conducta entre el pretest y el seguimiento a siete años 
entre postest y seguimiento no es estadísticamente significativa, $\mathrm{t}(20)=1.19$, ns, y los problemas de conducta continúan elevados en comparación con el grupo de intervención. Cuando se realizan comparaciones intergrupo, no se encuentran diferencias entre el grupo de intervención y el de control en el pretest, como se señaló anteriormente, $\mathrm{F}(1 / 54)=.03$, ns, pero sí en el postest, $\mathrm{F}(1 / 54)=12.46$, p $<.001$, y en el seguimiento, $\mathrm{F}(1 / 53)=4.67, \mathrm{p}<.05$, en ambos casos con puntuaciones superiores en el grupo control.

\section{Comparación entre el grupo de intervención y el grupo control en el seguimiento: Dificultades comportamentales, funcionamiento psicosocial y consumo de drogas}

Una vez comprobado que 1) los grupos de intervención y control son comparables entre sí, al representar adecuadamente los grupos que formaron parte del estudio inicial; 2) los grupos evolucionaron de forma diferente a lo largo del tiempo en el marcador básico de problemas de conducta, el siguiente paso fue comparar los dos grupos en un amplio rango de variables indicadoras del ajuste psicológico de los adolescentes. Esto nos permitió analizar en qué medida el paso por el programa se relaciona con marcadores de bienestar y adaptación social, incluyendo el consumo de drogas.

Se analizaron, en particular, tres clusters de variables: 1) problemas comportamentales (externalizantes e internalizantes), 2) competencia y funcionamiento psicosocial (en el ámbito familar, escolar y grupal) y 3) consumo de drogas. Teniendo en cuenta la multicolinealidad de las variables, se realizaron análisis multivariados (MANOVA) considerando conjuntamente cada grupo de variables; cuando el análisis multivariado mostró diferencias significativas, se realizaron, posteriormente, análisis de varianza univariados. De acuer- do con la perspectiva multi-informante adoptada en este estudio, en cada análisis se incluyó, para aquellos indicadores donde es pertinente, la información proporcionada por los padres y por los propios jóvenes.

Los resultados de la comparación entre el grupo de intervención y en grupo control se presentan a continuación.

Comparación en problemas comportamentales.

Para la comparación en las medidas de problemas comportamentales, se tomaron, por una parte, los indicadores de problemas generales de conducta y, por otra parte, como una categoría específica de problemas de conducta se consideraron las conductas agresivas (reactivas y proactivas).

En la Tabla 1 se presentan los resultados correspondientes a la comparación del grupo de intervención y el grupo control en las diversas medidas globales de problemas de comportamiento.

Los resultados del análisis multivariable indicaron que existen diferencias significativas en este grupo de variables. Específicamente, los análisis univariados mostraron que los chicos que formaron parte de la intervención presentan, siete años más tarde, menores niveles de problemas externalizantes y disruptivos, informados por los padres, menos impulsividad y menos conductas oposicionistas-desafiantes. No existen diferencias, sin embargo, en los problemas atencionales, ni en la conducta antisocial autoinformada. Tampoco existen diferencias en los problemas internalizantes.

Cuando se analizan, específicamente, las conductas agresivas, incluyendo medidas de agresión proactiva (meditada, instrumental) y reactiva (hostil, emocional), tanto informadas por padres como por chicos, el análisis multivariable mostró que no existen diferencias significativas entre ambos grupos $(\mathrm{F}=0.59,4 / 49$, n.s. $)$.

Tabla 1. Análisis multivariable de la varianza para la comparación entre el grupo de intervención y el grupo control en las medidas de problemas de conducta (seguimiento a siete años).

\begin{tabular}{|c|c|c|c|c|c|}
\hline & $\begin{array}{l}\text { Grupo Intervención } \\
\mathrm{N}=37 \\
\text { Media (SD) }\end{array}$ & $\begin{array}{l}\text { Grupo Control } \\
\mathrm{N}=21 \\
\text { Media (SD) }\end{array}$ & $\lambda$ & $F(g l)$ & $\eta^{2}$ \\
\hline & & & 0.65 & $3.64(6 / 42)^{\star *}$ & 0.34 \\
\hline CBCL-Externalizante (Inf. Padres) & $12.86(8.36)$ & $21.20(10.36)$ & & $8.91(1 / 47)^{\star \star}$ & 0.16 \\
\hline CBCL-Internalizante (Inf. Padres) & $12.80(8.36)$ & $9.18(5.96)$ & & $2.46(1 / 47)$ & 0.05 \\
\hline Falta de atención (Inf. Padres) & $12.76(7.96)$ & $14.00(7.17)$ & & $0.26(1 / 47)$ & 0.05 \\
\hline Hiperactividad/Impulsividad (Inf. Padres) & $6.69(4.86)$ & $9.66(4.46)$ & & $4.06(1 / 47)^{\star}$ & 0.08 \\
\hline Conductas oposicionistas-desafiantes (Inf. Padres) & $6.11(5.55)$ & $11.20(5.10)$ & & $9.14(1 / 47)^{\star \star}$ & 0.16 \\
\hline Conducta antisocial autoinformada & $9.09(9.21)$ & $12.66(11.48)$ & & $1.33(1 / 47)$ & 0.02 \\
\hline
\end{tabular}

Nota. ${ }^{\star}$ p $<.05 ;{ }^{\star \star}$ p $<.01$ 
Tabla 2. Análisis multivariable de la varianza para la comparación entre el grupo de intervención y el grupo control en las medidas de competencia psicosocial (seguimiento a siete años).

\begin{tabular}{llllll}
\hline & $\begin{array}{l}\text { Grupo Intervención } \\
\mathrm{N}=37\end{array}$ & $\begin{array}{l}\text { Grupo Control } \\
\mathrm{N}=21\end{array}$ & $\lambda$ & $\mathbf{F ( g l )}$ & $\mathbf{n}^{\mathbf{2}}$ \\
& Media (SD) & Media (SD) & & & \\
\hline Habilidades de regulación emocional (Inf. Padres) & $10.99(4.42)$ & $8.85(3.85)$ & 0.81 & $3.83(3 / 51)^{\star}$ & 0.18 \\
\hline Habilidades prosociales/comunicativas (Inf. Padres) & $15.91(4.46)$ & $11.45(5.09)$ & $3.26(1 / 53)$ & 0.05 \\
\hline Empatía (autoinformada) & $7.26(2.36)$ & $6.20(2.23)$ & $11.46(1 / 53)^{\star \star}$ & 0.17 \\
\hline
\end{tabular}

Nota. ${ }^{\star} \mathrm{p}<.05 ;{ }^{\star \star} \mathrm{p}<.01$

Tabla 3. Análisis multivariable de la varianza para la comparación entre el grupo de intervención y el grupo control en las medidas relativas a los iguales (seguimiento a siete años).

\begin{tabular}{|c|c|c|c|c|c|}
\hline & $\begin{array}{l}\text { Grupo Intervención } \\
\mathrm{N}=37 \\
\text { Media (SD) }\end{array}$ & $\begin{array}{l}\text { Grupo Control } \\
\mathrm{N}=21 \\
\text { Media (SD) }\end{array}$ & $\lambda$ & $F(g l)$ & $n^{2}$ \\
\hline & & & 0.22 & $3.47(3 / 41)^{\star}$ & 0.22 \\
\hline Número de amigos & $7.04(5.79)$ & $8.00(6.56)$ & & $1.00(1 / 40)$ & 0.00 \\
\hline Confianza en los amigos & $3.82(0.95)$ & $4.13(0.68)$ & & $0.77(1 / 39)$ & 0.02 \\
\hline Conducta antisocial de los amigos & $1.53(1.52)$ & $3.50(2.93)$ & & $7.79(1 / 40)^{\star \star}$ & 0.17 \\
\hline
\end{tabular}

Nota. ${ }^{\star}$ p $<.05 ;{ }^{* \star}$ p $<.01$

Comparaciones en competencias y funcionamiento psicosocial.

El análisis de variables en este ámbito se realizó considerando, por una parte las competencias psicosociales, por otro el funcionamiento escolar y, por otro, el funcionamiento social en el grupo de amigos.

En la Tabla 2 se presentan los resultados de la comparación entre ambos grupos atendiendo a competencias psicosociales: habilidades de regulación emocional, habilidades prosociales/comunicativas y empatía (autoinformada).

El análisis multivariable reveló diferencias significativas en este grupo de variables. Las diferencias se sitúan, particularmente, en las habilidades prosociales/comunicativas, donde el grupo de intervención puntúa más alto que el control.

Cuando se examina el funcionamiento escolar (implicación escolar, absentismo, rendimiento académico), el contraste multivariado no fue significativo ( $\mathrm{F}=1.77,3 / 52$, n.s. $)$, aunque el contraste univariado de absentismo sí indica que las faltas injustificadas del grupo control son significativamente más frecuentes que las del grupo de tratamiento $(\mathrm{F}=5.01,1 / 54, \mathrm{p}<.05)$.

En la Tabla 3 se presentan los resultados correspondientes a las variables del grupo de amigos.

La Tabla 3 muestra que ambos grupos difieren también en este grupo de variables, y que las diferencias se establecen, particularmente, en la conducta antisocial de los amigos, mayor en el grupo control que en el grupo de intervención.

Comparaciones en consumo de drogas.

Para los análisis multivariables, las variables relacionadas con el consumo de drogas fueron analizadas agrupadas en los siguientes grupos: actitudes e intenciones, edad de inicio de consumo, frecuencias de consumo y cantidad de consumo de alcohol.

En primer lugar, se analizaron las actitudes e intenciones respecto al consumo de drogas. La Escala de Actitudes hacia las Drogas forma parte del Cuestionario de Consumo de Drogas (CCD; Luengo et al., 1999), y evalúa en qué medida los adolescentes mantienen una valoración positiva del consumo de drogas y de sus efectos (e.g., "Fumar porros es una experiencia agradable que hay que vivir", "El consumo de alcohol me perjudicaría en mis estudios", "Se deben probar las drogas para poder hablar a los demás sobre sus efectos", "Tener alcohol hace las fiestas más divertidas"). La Escala de Intenciones pregunta, concretamente, con qué probabilidad el joven cree que consumiría drogas (tabaco, alcohol, cannabis) el próximo fin de semana, si tuviese la oportunidad ("Seguro que no", "Probablemente no", "Probablemente sî", "Seguro que sî”; estas respuestas se puntúan de 0 a 3). Los resultados se presentan en la Tabla 4. 
Tabla 4. Análisis multivariable de la varianza para la comparación entre el grupo de intervención y el grupo control en las actitudes e intención de consumir drogas (seguimiento a siete años).

\begin{tabular}{|c|c|c|c|c|c|}
\hline & $\begin{array}{l}\text { Grupo Intervención } \\
\mathrm{N}=37\end{array}$ & $\begin{array}{l}\text { Grupo Control } \\
\mathrm{N}=21\end{array}$ & $\lambda$ & $F(g l)$ & $\eta^{2}$ \\
\hline & Media (SD) & Media (SD) & & & \\
\hline & & & 0.77 & $3.61(4 / 51)^{\star}$ & 0.83 \\
\hline Actitudes hacia las drogas & $6.46(3.69)$ & $10.30(4.08)$ & & $12.87(1 / 54)^{\star \star}$ & 0.19 \\
\hline Intención tabaco & $0.40(0.87)$ & $1.29(1.30)$ & & $9.47(1 / 54)^{\star \star}$ & 0.14 \\
\hline Intención alcohol & $0.77(1.06)$ & $1.48(1.16)$ & & $5.22(1 / 54)^{\star}$ & 0.08 \\
\hline Intención cannabis & $0.26(0.61)$ & $0.67(1.01)$ & & $3.56(1 / 54)$ & 0.06 \\
\hline
\end{tabular}

Nota. ${ }^{\star}$ p $<.05 ;{ }^{\star \star}$ p $<.01$

Tabla 5. Análisis multivariable de la varianza para la comparación entre el grupo de intervención y el grupo control en la frecuencia de consumo de tabaco (seguimiento a siete años).

\begin{tabular}{llllll}
\hline & $\begin{array}{l}\text { Grupo Intervención } \\
\mathrm{N}=37\end{array}$ & $\begin{array}{l}\text { Grupo Control } \\
\mathrm{N}=21\end{array}$ & $\lambda$ & $\mathbf{F ( g l )}$ & $\mathbf{\eta}^{\mathbf{2}}$ \\
& Media (SD) & Media (SD) & & & \\
\hline Frecuencia tabaco mes & & & .71 & $7.94(2 / 39)^{\star \star}$ & 0.28 \\
\hline Frecuencia tabaco vida & $0.59(1.50)$ & $2.67(2.19)$ & $13.18(1 / 40)^{\star \star}$ & 0.24 \\
\hline
\end{tabular}

Nota. ${ }^{* \star}$ p $<.01$

El análisis multivariable mostró una F significativa y, particularmente, se encuentran diferencias en actitudes (más favorables en el grupo control) y en la intención de consumir tabaco y alcohol (más alta también en el grupo control). Cuando se analizan la proporción de chicos que "probablemente sî" si consumirían tabaco, se encuentra que un $11 \%$ en el grupo de intervención estarían dispuestos a consumir, frente a un $42 \%$ en el grupo control (chi-cuadrado: 7.59, 1 gl, p<.001). En cuanto a los chicos que "probablemente sí" consumirían alcohol, un $11 \%$ del grupo de intervención elige esta respuesta, frente al $35 \%$ del grupo control (chi-cuadrado: 6.23, $1 \mathrm{gl}, \mathrm{p}<.01$ ).

No se encuentran, sin embargo, diferencias en las edades de inicio ( $\mathrm{F}$ multivariada=0.47, 2/29 gl, ns), que fueron de 13.00 (intervención) y 13.77 (control) para tabaco; 14.18 (intervención) y 13.27 (control) para alcohol; y 14.63 (intervención) y 14.40 (control) para cannabis.

Se analizaron también las frecuencias de consumo en el último mes y a lo largo de la vida: e.g. “¿Cuántas veces has fumado cigarrillos en el último mes?”; “Cuántas veces has tomado más de un sorbo de alcohol a lo largo de toda tu vida?". Las opciones de respuesta se sitúan entre "Nunca" (con valor 0) y "Más de 20" (con valor 5). El análisis de comparación entre medias no mostró diferencias significativas entre el grupo de intervención y el control en cuanto al consumo de alcohol ( $\mathrm{F}$ multivariada $=1.09,2 / 49$, n.s.) ni al de cannabis ( $\mathrm{F}$ multivariada=1.84, 2/38, n.s.), si bien en ambos casos las medias tienden a ser más altas en el grupo control que en el de intervención. Sí se observan diferencias significativas, sin embargo, en la frecuencia de consumo de tabaco. Los resultados se presentan en la Tabla 5 .

En ambas medidas de frecuencia se obtuvieron diferencias significativas. El grupo control muestra frecuencias de consumo de tabaco superiores a las del grupo de intervención. Cuando se examinan estos datos datos desde un punto de vista categorial, se encuentra que el $33 \%$ de los chicos en el grupo de intervención afirman haber consumido tabaco más de una vez en su vida, frente a a un $75 \%$ de los chicos del grupo control (chi-cuadrado=6.98, 1 gl, p<.001). Así mismo, el $11 \%$ de los chicos del grupo de tratamiento manifiestan haber consumido tabaco en el último mes, frente a un $56 \%$ en el grupo control (chi-cuadrado $=13.42,1$ gl, $\mathrm{p}<.001$ ).

Finalmente, se observaron diferencias en las medidas de cantidad de consumo de alcohol (“¿Cuántas consumiciones sueles tomar cuando bebes?”, “¿Cuántas veces te has emborrachado en el último año?”). Los resultados se presentan en la Tabla 6 .

Cuando se analizan los datos desde un punto de vista categorial, se encuentra que el $26 \%$ de los chicos en el grupo de tratamiento afirman que suelen consumir más de una consumición cuando beben, frente al $62 \%$ de los chicos en el grupo control (chi-cuadrado=5.20, 1 gl, p<.05). En cuanto al número de borracheras en el último año, un $19 \%$ de los chicos en el grupo de tratamiento afirma haberse emborrachado, frente a un $56 \%$ en el grupo control (chi-cuadra$\mathrm{do}=6.10,1 \mathrm{gl}, \mathrm{p}<.05)$. 
Tabla 6. Análisis multivariable de la varianza para la comparación entre el grupo de intervención y el grupo control en la intensidad del consumo de alcohol (seguimiento a siete años).

\begin{tabular}{llllll}
\hline & $\begin{array}{l}\text { Grupo Intervención } \\
\mathrm{N}=37\end{array}$ & $\begin{array}{l}\text { Grupo Control } \\
\mathrm{N}=21\end{array}$ & $\lambda$ & $\mathbf{F ( g l )}$ & $\boldsymbol{\eta}^{\mathbf{2}}$ \\
& Media (SD) & Media (SD) & & & \\
\hline & & & .77 & $5.53(2 / 38)^{\star \star}$ & 0.22 \\
\hline Número consumiciones & $0.86(1.08)$ & $1.87(0.83)$ & $9.87(1 / 39)^{\star \star}$ & 0.30 \\
\hline Número borracheras & $0.19(0.40)$ & $0.60(0.63)$ & $6.39(1 / 39)^{\star \star}$ & 0.14 \\
\hline
\end{tabular}

Nota. ${ }^{\star \star}$ p $<.001$

\section{Discusión}

La necesidad de desarrollar programas de prevención indicada de corte multicomponente ha sido puesta de relieve repetidamente en los últimos años (Boxmeyer, Lochman, Powell, y Powe, 2015). La identificación de trayectorias desfavorables, asociadas a problemas de conducta tempranos, ha impulsado la propuesta de programas dirigido a niños, con el fin de prevenir múltiples disfunciones psicológicas y sociales, incluyendo patrones severos de consumo de drogas. Este trabajo ha permitido comprobar la eficacia, a largo plazo, de un programa multicomponente dirigido a niños con problemas de conducta, a sus padres y a sus profesores.

Como hemos visto, la trayectoria de evolución delos problemas de conducta difiere en el grupo de intervención respecto al control.

En el grupo control los problemas se mantienen lo largo del tiempo sin diferencias significativas entre mediciones, con una tendencia descedente emtre el postest y el seguimiento, congruente con los resultados de otros estudios que han examinado la evolución de los problemas de conducta informados por los padres desde la niñez (Anselmi et al., 2008; Hofstra, Van der Ende y Verhulst, 2000). El grupo de intervencion, sin embargo, presenta descensos con el programa, que se mantienen a lo largo del tiempo. Así, siete años después del comienzo de la intervención, se comprueba que el programa multicomponente reduce los problemas de comportamiento perturbadores en la adolescencia, particularmente los comportamiento de carácter impulsivo y oposicionista. Es necesario señalar, no osbstante, que los efectos aparecen claramente cuando se utilizan los informes de los padres, y no tanto cuando los problemas son informados por los chicos. Es posible que, dado que el programa tiene en la intervención familiar un centro prioritario, los efectos positivos del programa sean percibidos con más nitidez en el contexto de las interacciones padres-hijos. Por otra parte, es llamativo que no se aprecien efectos significativos sobre las conductas agresivas, a pesar de que el enfrentamiento a la ira es uno de los contenidos más enfatizados dentro del componente dirigido a niños. El programa parece tener un efecto más generalizado sobre conductas impulsivas y de desafío a las normas, pero, sin embargo, los componentes dirigidos específicamente a la reducción de la agresión no tienen el impacto esperado a largo plazo. En relación con lo anterior, es llamativo también que no se encuentren diferencias significativas en las habilidades de regulación emocional ni en la empatía, lo cual sugiere que los efectos específicos sobre las emociones de corte interpersonal se atenúan a lo largo del tiempo y, por ello, estos componentes deberían ser reforzados en la intervención.

En general, los efectos significativos sobre los problemas de conducta a largo plazo son concordantes con otros programas multicomponente que también han constatado un efecto preventivo a largo plazo sobre problemas externalizantes (Eddy et al., 2003; Hektner, August, Bloomquist, Lee y Kimes-Dougan, 2014; Webster-Stratton, Reid y Hammond, 2001) y sobre delincuencia (Conduct Problems Prevention Research Group, 2010; Tremblay, Pagani-Kurtz, Masse, Vitaro y Pihl, 1995).

Por otra parte, los resultados muestran que el programa es capaz de afectar favorablemente a las habilidades prosociales y de comunicación, y disminuye la implicación con amigos antisociales en la adolescencia. Dada la relevancia que estos factores presentan dentro de los modelos de cronificación de los problemas de conducta (Dodge et al., 2010), este es un resultado que puede considerarse también favorable para la prevención de patrones de comportamiento antisocial persistente.

Cuando, de un modo específico, se examinan los efectos sobre el consumo de drogas, se encuentra evidencia también de efectos significativos. La participación en el programa se asocia con actitudes más desfavorables ante las drogas y menor intención de consumo de tabaco y alcohol. También se observan efectos significativos en la prevención del consumo de tabaco, con una frecuencia menor de consumo por parte de los chicos que participaron en el programa, tanto a lo largo de la vida, como en el último mes. Por lo que respecta al alcohol, no parece afectada la frecuencia de consumo (que, de hecho, es alta en la población general de adolescentes de estas edades; Plan Nacional sobre Drogas, 2013), pero sí la cantidad de consumo, con 
un menor número de consumiciones y de borracheras en el grupo de intervención. Este resultado es especialmente destacable, dado que el aumento en la cantidad en el consumo de alcohol es un parámetro especialmente preocupante en las sociedades occidentales, de acuerdo con los estudios epidemiológicos de la última década (ESPAD, 2011; Johnston, O’Malley, Miech, Bachman y Schulenberg, 2015). No se encuentran efectos significativos sobre el consumo de cannabis; no obstante, dado que el consumo de cannabis tiene, en general, un patrón de iniciación más tardío que el consumo de tabaco y alcohol, un seguimiento a más largo plazo sería necesario para valorar adecuadamente el efecto sobre los patrones de consumo de esta sustancia. El consumo de cannabis, de hecho, se ha asociado a consumo de alcohol y de tabaco en jóvenes españoles (Miguez Varela y Becoña, 2015), por lo que sería relevante examinar si, en seguimientos más amplios, emergen también efectos en relación con el cannabis. Por lo demás, los resultados sobre el consumo de drogas están en línea con algunos trabajos previos (Eddy et al., 2003; Zonnevyille et al., 2007) que también han proporcionado evidencia de los efectos sobre el consumo de drogas, y específicamente sobre el consumo de alcohol (Eddy et al., 2003), de programas multicomponente enfocados en el tratamiento de los problemas de conducta externalizante.

Así pues, este estudio contribuye a afianzar la utilidad de los programas multicomponente fundamentados en los modelos de la psicopatología del deasarrollo para ejercer efectos sobre las actitudes y el comportamiento de los chicos cuando estos se adentran en la adolescencia. El programa evaluado en este estudio, que incluye una intervención integrada y coordinada sobre los padres, los profesores y los propios niños, muestra efectos sobre múltiples indicadores del ajuste psicosocial, incluyendo el consumo de drogas.

Además de las implicaciones que estos resultados pueden tener para orientar la prevención indicada dirigida a niños, estos resultados proporcionan también apoyo a los modelos que están en la base de estos programas. Como han señalado otros autores (LeMarquand, Tremblay y Vitaro, 2001), comprobar la eficacia de los programas de intervención permite validar los principios de partida sobre el origen y el mantenimiento de los problemas sobre los que se interviene. En nuestro caso, la importancia de los problemas de conducta en el desarrollo del consumo de drogas queda respaldada por los efectos de la intervención.

Este estudio proporciona así nuevos datos de apoyo sobre la eficacia de un tipo de programas cuyo impacto a largo plazo necesita ser sistemáticamente examinado. Además, la utilización de múltiples informantes proporciona solidez a los efectos encontrados. El estudio, con todo, presenta limitaciones que la investigación futura habrá de superar. Por una parte, el limitado tamaño muestral (usual en este tipo de estudios con niños en alto riesgo; Zonnevylle-Bender et al., 2007) resta poder estadístico a los análisis realizados.
En este sentido, la realización de estudio multicéntricos permitirá ampliar el alcance de los estudio de eficacia; esto permitirá además incoporar el estudio de los moderadores de la eficacia, para comprender qué características de los niños, de su entorno, o de las condiciones de aplicación permiten maximizar los efectos positivos de la intervención. Variables como el género, estatus socioeconómico de la familia, severidad de los problemas de conducta iniciales, comorbilidades, así como fidelidad e integridad de implementación son algunos de los elementos que habrán de ser investigados; estos factores han sido identificados, en las últimas décadas, como factores que afectan al éxito de los programas de entrenamiento de padres (Maughan, Christiansen, Jenson, Olympia y Clark, 2005; Robles y Romero, 2011) y podrían ser estudiados también como moderadores en los programas como este. Particularmente, el papel de las condiciones contextuales como variables moderadoras merece ser sistemáticamente examinado, dada la relevancia que han mostrado en estudios sobre conductas adictivas los factores relacionados con el entorno macrosocial (Buil, Solé Moratilla y García Ruiz, 2015) y familiar (Hernández-Serrano, Font-Mayolas y Gras, 2015). La ampliación del tamaño muestral permitirá además desgranar los mecanismos de influencia del programa, y examinar qué componentes del programa resultan críticos para sus efectos positivos.

Otro aspecto que deberá ser objeto de examen, dados los costes asociados a este tipo de programas, es su eficiencia, en términos de costes y beneficios. Algunas revisiones previas han proporcionado apoyo a la eficiencia de otros programas multicomponente (Foster et al., 2007), aunque este es un aspecto que deberá ser sistemáticamente investigado a medida que se vayan desarrollando estudios a largo plazo.

De momento, la evidencia proveniente de este estudio proporciona apoyo a la inclusión de los programas de reducción de problemas de conducta tempranos como programas para la prevención del abuso de drogas (Glantz, 2002). Se ha dicho, en este sentido, que la intervención multicomponente podría tener también un efecto "cascada”, generando una cadena de cambios positivos en la relación del individuo con el ambiente (Patterson, Forgatch y Desarmo, 2010), capaz de alterar sustancialmente la trayectoria de desarrollo del individuo, y particularmente, el riesgo de implicación en un consumo abusivo de drogas. La intervención a edades tempranas, por otra parte, es compatible con la investigación neurocientífica, que apoya la importancia de los programas de desarrollo de las funciones cognitivas de autorregulación y autocontrol cuando los sistemas neurales correspondientes tienen aún una alta plasticidad y son responsivos a los inputs del ambiente (véase Fishbein y Tarter, 2009). De hecho, la evidencia que se empieza a generar sobre la relevancia de los problemas de conducta ha llevado a afirmar que los problemas de conducta podrían ofrecer "la más grande" oportunidad para prevención en el ámbito de la salud mental (Harley, Murtagh y Cannon, 2008). 


\section{Reconocimientos}

Esta investigación pudo ser realizada gracias a las subvenciones recibidas por parte de la Delegación del Gobierno para el Plan Nacional de Drogas (2012I024) y de la Dirección General de Investigación (PSI2015-65766-R, MINECO/FEDER).

\section{Conflicto de intereses}

Los autores no tienen conflictos de intereses que declarar.

\section{Referencias}

Achenbach, T. M. (1991a). Manual for the Teacher's Report Form and 1991 Profile. Burlington: University of Vermont. Achenbach, T. M. (1991b). Manual for the Child Behavior Checklist and 1991 Profile. Burlington: University of Vermont.

Anselmi, L., Barros, F.C., Teodoro, M.L., Piccinini, C.A., Menezes, A.M.B., Araujo, C.L. y Rohde, L.A. (2008). Continuity of behavioral and emotional problems from pre-school years to pre-adolescence in a developing country. Journal of Child Psychology and Psychiatry, 49, 499-507.

Armsden, G. C. y Greenberg, M. T. (1987). The Inventory of Parent and Peer Attachment: Relationships to well-being in adolescence. Journal of Youth and Adolescence, 16, 427-454.

Barkley, R.A. (1997). Defiant Children: A Clinician's Manual for Assessment and Parent Training ( $2^{\circ}$ ed.). Nueva York: Guilford.

Berry, J. W., Phinney, J. S., Sam, D. y Vedder, P. (Eds.) (2006). Immigrant youth in cultural Transition: Acculturation, identity and adaptation across national contexts. Hillsdale: Erlbaum.

Boxmeyer, C.L., Lochman, J.E., Powell, N.P. y Powe, C.E. (2015). Preventing conduct disorders and related problems. En L.M. Scheier (Ed.), Handbook of Adolescent Drug Use Prevention: Research, Intervention Strategies, and Practice (pp. 125-134). Washington, DC: American Psychological Association.

Buil, P., Solé Moratilla, M.J. y García Ruiz, P. (2015). Online gambling advertising in Spain: A study on the protection of minors. Adicciones, 27, 198-204.

Cicchetti, D. y Cohen, D.J. (2006). Developmental Psychopathology. Nueva York: Wiley.

Conduct Problems Prevention Research Group (1995). Social Competence Scale (Parent Version). University Park, PA: Pennsylvania State University.

Conduct Problem Prevention Research Group (2004). The effects of the Fast Track program on serious problem outcomes at the end of elementary school. Journal of Child Psychology and Psychiatry and Allied Disciplines, 36, 1141-1159.
Conduct Problems Prevention Research Group (2010). Fast Track intervention effects on youth arrests and delinquency. Journal of Experimental Criminology, 6, 131-157.

Del Barrio, V., Aluja, A., y García, L.F. (2004). Relationship between empathy and the Big Five personality traits in a sample of Spanish adolescents. Social Behavior and Personality, 32, 677-682.

Dodge, K. A. y Coie, J. D. (1987). Social information processing factors in reactive and proactive aggression in children's peer groups. Journal of Personality and Social Psychology, 53, 1146-1158.

Dodge, K.A., Malone, P. S., Lansford, J.E., Miller, S., Pettit, G.S. y Bates, J.E. (2010). A dynamic cascade model of the development of substance use onset. Nueva York: Wiley.

Eddy, J.M., Reid, J.B., Stoolmiller, M. y Petrow, R.A. (2003). Outcomes during middle school for an elementary school-based preventive intervention for conduct problems: Follow-up results from a randomized trial. Behavior Therapy, 34, 535-582.

EMCDDA (2009). Preventing later substance use disorders in at-risk children and adolescents: A review of the theory and evidence base of indicated prevention. Lisboa: European Monitoring Center for Drug and Drug Addiction.

ESPAD (2011). The 2011 ESPAD Report: Substance use among students in 36 European countries. Estocolmo: The Swedish Council for Information on Alcohol and Other Drugs.

Fishbein, D. y Tarter, R. (2009). Infusing neuroscience into the study and prevention of drug misuse and co-ocurring aggressive behavior. Substance Use and Misuse, 44, 1204-1235.

Foster, E. M., Olchowski, A.E. y Webster-Stratton, C.H. (2007). Is stacking intervention components cost-effective? An analysis of the Incredible Years program. Journal of the American Academy of Child and Adolescent Psychiatry, $46,1414-1424$.

Foxcroft, D.R. (2014). "Form ever follows function. This is the law". A prevention taxonomy based on a functional typology. Adicciones, 26, 10-14.

Glantz, M.D. (2002). Introduction to the special issue on the impact of childhood psychopathology interventions on subsequent substance abuse: Pieces of the puzzle. Journal of Consulting and Clinical Psychology, 70, 12031206.

Gordon, R. S. (1983). An operational classification of disease prevention. Public Health Reports, 98, 107-109.

Granic, I. y Patterson, G.R. (2006). Toward a comprehensive model of antisocial development: A dynamic systems approach. Psychological Review, 113, 101-131.

Haller, M., Handley, E., Chassin, L. y Bountress (2010). Developmental cascades: Linking adolescent substance use, affiliation with substance use promoting peers, and academic achievement to adult substance use disorders. Development and Psychopathology, 22, 899-915. 
Harley, M., Murtagh, A. y Cannon, M. (2008). Conduct disorder: Psychiatry's greatest opportunity for prevention. Psychological Medicine, 38, 929-931.

Hawkins, E.H. (2009). A tale of two systems: Co-occurring mental health and substance abuse disorders treatment for adolescents. Annual Review of Psychology, 60, 197-227.

Hektner, J.M., August, G.J., Bloomquist, M.L., Lee,S. y Klimes-Dougan, B.(2014). A 10-year randomized controlled trial of the Early Risers conduct problems preventive intervention: Effects on externalizing and internalizing in late high school. Journal of Consulting and Clinical Psychology, 82, 355-360.

Hernández-Serrano, O., Font-Mayolas, S. y Gras, M.E. (2015). Polydrug use and its relationship with the familiar and social context amongst young college students. Adicciones, 27, 205-213.

Hofstra, M.B., Van der Ende, J. y Verhulst, F.C. (2000). Continuity and change of psychopathology from childhood into adulthood: A 14-year follow-up study. Journal of the American Academy of Child and Adolescent Psychiatry, 39, 850-858.

Hser, Y.I., Grella, C.E., Collins, C. y Teruya, C. (2003). Druguse initiation and conduct disorder among adolescents in drug treatment. Journal of Adolescence, 26, 331-345.

Johnston, L. D., O’Malley, P. M., Miech, R. A., Bachman, J. G., y Schulenberg, J. E. (2015). Monitoring the Future National Survey results on drug use: 1975-2014: Overview, key findings on adolescent drug use. Ann Arbor: Institute for Social Research, The University of Michigan.

Larson, J. y Lochman, J.E. (2002). Helping schoolchildren cope with anger. A cognitive-behavioral intervention. Nueva York: Guilford.

LeMarquand, D., Tremblay, R.E. y Vitaro, F. (2001). The prevention of conduct disorder: A review of successful and unsuccessful experiments. En J. Hill y B. Maughan (Eds.), Conduct Disorders in Childhood and Adolescence (pp. 449-478). Cambridge: Cambridge University Press.

López-Romero, L., Romero, E., y Andershed, H. (2015). Conduct problems in childhood and adolescence: Developmental trajectories, predictors and outcomes in a sixyear follow-up. Child Psychiatry and Human Development, 46, 762-773

Luengo, M.A., Otero, J.M., Romero, E., Gómez-Fraguela, X.A. y Tavares, E.T. (1999). Análisis de items para la evaluación de la conducta antisocial: Un estudio transcultural. Revista Iberoamericana de Diagnóstico y Evaluación Psicológica, 1, 21-36.

Luengo, M.A., Romero, E., Gómez-Fraguela, J.A., Garra, A. y Lence, M. (1999). La prevención del consumo de drogas y la conducta antisocial en la escuela: Análisis y evaluación de un Programa. Madrid: Ministerio de Educación y Cultura, Ministerio de Sanidad y Consumo y Ministerio del Interior.

Luengo, M.A., Villar, P., Sobral., J., Romero, E. y Gómez-Fraguela, X.A. (2009). El consumo de drogas en los adolescentes inmigrantes: Implicaciones para la prevención. Revista Española de Drogodependencias, 3, 420-447.

Martel, M.M., Pierce, L., Nigg, J.T., Jester, J.M., Adams. K., Puttler, L.I,....Zucker, R.A. (2009). Temperament pathways to childhood disruptive behavior and adolescent substance abuse: Testing a cascade model. Journal of $A b$ normal Child Psychology, 37, 363-373.

Maughan, D. R., Christiansen, E., Jenson, W. R., Olympia, D., y Clark, E. (2005). Behavioral parent training as a treatment for externalizing behaviors and disruptive behavior disorders: A meta-analysis. School Psychology Review, 34, 267-286.

Míguez Varela, M.C. y Becoña, E. (2015). Do cigarette smoking and alcohol consumption associate with cannabis use and problem gambling among Spanish adolescents? Adicciones, 27, 8-16.

NIDA (2003). Preventing drug use among children and adolescents: A research-based guide. Bethseda, MD: US Department of Health and Human Services.

Patterson, G.R., Forgatch, M.S. y Desarmo, D.S. (2010). Cascading effects following intervention. Development and Psychopathology, 22, 949-970.

Plan Nacional sobre Drogas (2013). Encuesta Escolar sobre Uso de Drogas en Estudiantes de Enseñanzas Secundarias (ES TUDES) 2012-2013. Madrid: Plan Nacional sobre Drogas.

Robles, Z. (2009). Intervención sobre problemas de conducta de inicio temprano. Evaluación de un programa de entrenamiento para padres. Tesis doctoral. Universidad de Santiago de Compostela.

Robles, Z. y Romero, E. (2011). Programas de entrenamiento para padres de niños con problemas de conducta: Una revisión de su eficacia. Anales de Psicología, 27, 86-101.

Romero, E., Robles, Z., y Lorenzo, E. (2006). Prácticas parentales, atmósfera familiar y problemas de conducta externalizante en la infancia. Revista de Psiquiatría, 33, 84-92.

Romero, E., Villar, P., Luengo, M.A. y Gómez-Fraguela, J. A. (2009). EmPeCemos: Un programa multicomponente para la prevención indicada de los problemas de conducta y el abuso de drogas. Revista Española de Drogodependencias, 4, 420-447

Romero, E., Villar, P., Luengo, M.A., Gómez-Fraguela, X.A. y Robles, Z. (2014). EmPeCemos. Programa para la Intervención sobre los Problemas de Conducta infantiles. Madrid: TEA.

Romero, E., Villar, P. y Gómez-Fraguela, X.A. (2010). Intervening on family, school and children for prevention of early-onset conduct problems. European Journal of Public Health, 20 (Suplemento 1: Proceedings from the 3rd European Public Health Conference), 90.

Salvo, N., Bennett, K., Cheung, A., Chen, Yo., Rice,M.,, Rush, B., ... y Bowlby, A. (2012). Prevention of substance use in children/adolescents with mental disorders: a 
systematic review. Journal of the Canadian Academy of Child and Adolescent Psychiatry, 21, 245-252.

Scheier, L.M. (Ed.) (2015). Handbook of adolescent drug use prevention: Research, intervention Strategies, and practice. Washington, DC: American Psychological Association.

Sitnick, S.L., Shaw, D.S. y Hyde, L.W. (2014). Precursors of adolescent substance use from early childhood and early adolescence: Testing a developmental cascade model. Development and Psychopathology, 26, 125-140.

Sobral, J., Gómez-Fraguela, X.A., Romero, E., Luengo, M.A. y Villar, P. (2012). Riesgo y protección de desviación social en adolescentes inmigrantes: Personalidad, familia y aculturación. Anales de Psicología, 28, 664-674.

Thornberry, T.P., Lizotte, A.J., Krohn, M.D., Farnworth, M. y Jang, S.J. (1994). Delinquent peers, beliefs, and delinquent behavior: A longitudinal test of interactional theory. Criminology, 32, 47-83.

Tremblay, R.E., Pagani-Kurtz, L., Masse, L.C.,Vitaro, F.y Pihl, R.O. (1995). A bimodal preventive intervention for disruptive kindergarten boys: Its impact through mid-adolescence. Journal of Consulting and Clinical Psychology, 63, 560-568.

Webster-Stratton, C. y Hammond, M. (1997). Treating children with early-onset conduct problems: A comparison of child and parent training interventions. Journal of Consulting and Clinical Psychology, 65, 93-109.

Webster-Stratton, C., Reid, M.J. y Hammond, M. (2001). Preventing conduct problems, promoting social competence: A parent and teacher training partnership in Head Start. Journal of Clinical Child Psychology, 30, 283302.

Zonnevylle-Bender, M.J.S., Matthys, W., Van De Wiel, N.M.H. y Lochman, J.E. (2007). Preventive effects of treatment of disruptive behavior disorder in middle childhood on substance use and delinquent behavior. Journal of the American Academy of Child and Adolescent Psychiatry, 46, 33-39. 